Portland State University

PDXScholar

9-1-1993

\title{
Modeling complex ecological economic systems: toward an evolutionary, dynamic understanding of people and nature
}

\author{
Robert Costanza \\ Portland State University \\ Lisa Wainger \\ Carl Folke \\ Karl-Göran Mäler
}

Follow this and additional works at: https://pdxscholar.library.pdx.edu/iss_pub

Part of the Sustainability Commons

Let us know how access to this document benefits you.

\section{Citation Details}

Costanza, R., L. Wainger, C. Folke, and K-G Maler. 1993. Modeling complex ecological economic systems: toward an evolutionary, dynamic understanding of people and nature. BioScience 43:545-555.

This Article is brought to you for free and open access. It has been accepted for inclusion in Institute for Sustainable Solutions Publications and Presentations by an authorized administrator of PDXScholar. Please contact us if we can make this document more accessible: pdxscholar@pdx.edu. 


\title{
Modeling Complex Ecological Economic Systems
}

\author{
Toward an evolutionary, dynamic understanding of people \\ and nature
}

\author{
Robert Costanza, Lisa Wainger, Carl Folke, and Karl-Göran Mäler
}

$\mathbf{R}$ ecent understanding about system dynamics and predictability that has emerged from the study of complex systems is creating new tools for modeling interactions between anthropogenic and natural systems. A range of techniques has become available through advances in computer speed and accessibility and by implementing a broad, interdisciplinary systems view.

Systems are groups of interacting, interdependent parts linked together by exchanges of energy, matter, and information. Complex systems are characterized by strong (usually nonlinear) interactions between the parts, complex feedback loops that make it difficult to distinguish cause from effect, and significant time and space

Robert Costanza is director of the Maryland International Institute for Ecological Economics (MIIEE), Center for Environmental and Estuarine Studies (CEES), The University of Maryland System, Solomons, MD 20688-0038. He is also a professor in CEES and director of the complex systems research program of the Beijer International Institute of Ecological Economics (BIIEE) of the Royal Swedish Academy of Sciences. Lisa Wainger is a graduate research assistant at MIIEE, supported in part by a grant from BIIEE. Carl Folke is the deputy director of BIIEE, S-104 05 Stockholm, Sweden. He is also an assistant professor in the Department of Systems Ecology, Stockholm University, S-106 91 Stockholm, Sweden. KarlGöran Mäler is director of BIIEE. He is also a professor at the Stockholm School of Economics, S-113 83 Stockholm, Sweden. (C) 1993 American Institute of Biological Sciences.

A comprehensive
understanding of linked
systems requires the
synthesis and integration
of several different
conceptual frames

lags, discontinuities, thresholds, and limits. These characteristics all result in scientists' inability to simply add up or aggregate small-scale behavior to arrive at large-scale results (Rastetter et al. 1992, von Bertalanffy 1968). Ecological and economic systems both independently exhibit these characteristics of complex systems. Taken together, linked ecological economic systems are devilishly complex.

Although almost any subdivision of the universe can be thought of as a system, modelers of systems usually look for boundaries that minimize the interaction between the system under study and the rest of the universe in order to make their job easier. The interactions between ecological and economic systems are many and strong. So, splitting the world into separate economic and ecological systems is a poor choice of boundary.

Classical (or reductionist) scientific disciplines tend to dissect their subject into smaller and smaller isolated parts in an effort to reduce the problem to its essential elements. To allow the dissection of system components, it must be assumed that interactions and feedbacks between system elements are negligible or that the links are essentially linear so they can be added up to give the behavior of the whole (von Bertalanffy 1968). Complex systems violate the assumptions of reductionist techniques and therefore are not well understood using the perspective of classical science. In contrast, systems analysis is the scientific method applied across many disciplines, scales, resolutions, and system types in an integrative manner.

In economics, for example, a typical distinction is made between partial equilibrium analysis and general equilibrium analysis. In partial equilibrium analysis, a subsystem (a single market) is studied with the underlying assumption that there are no important feedback loops from other markets. In general equilibrium analysis, on the other hand, the totality of markets are studied to bring out the general interdependence in the economy. The large-scale, wholeeconomy, general equilibrium effects are usually quite different from the sum of the constituent small-scale partial equilibrium effects. Add to this observation the further complication that in reality equilibrium is never achieved, and one can begin to see the limitations of classical, reductionist science in understanding complex systems.

Economic and ecological analysis needs to shift away from implicit assumptions that eliminate links within and between economic and natural 
Table 1. The limits of analytical methods in solving mathematical problems (after von Bertalanffy 1968). The thick solid line divides the range of problems that are solvable with analytical methods from those that are difficult or impossible using analytical methods and require numerical methods and computers to solve. Systems problems are typically nonlinear and fall in the range that requires numerical methods. It should be noted that whereas some special problems that fall in the areas labeled impossible in the table are actually possible to solve using analytical methods (frequently requiring special tricks), in general one cannot depend on a solution being available. Computers have guaranteed that a solution can be found in all the cases listed in the table.

\begin{tabular}{|c|c|c|c|c|c|c|}
\hline \multirow[b]{2}{*}{ Equations } & \multicolumn{3}{|c|}{ Linear } & \multicolumn{3}{|c|}{ Nonlinear } \\
\hline & $\begin{array}{c}\text { One } \\
\text { equation }\end{array}$ & $\begin{array}{l}\text { Several } \\
\text { equations }\end{array}$ & $\begin{array}{c}\text { Many } \\
\text { equations }\end{array}$ & $\begin{array}{c}\text { One } \\
\text { equation }\end{array}$ & $\begin{array}{c}\text { Several } \\
\text { equations }\end{array}$ & $\begin{array}{c}\text { Many } \\
\text { equations }\end{array}$ \\
\hline Algebraic & Trivial & Easy & Difficult & Very difficult & Very difficult & Impossible \\
\hline Ordinary differential & Easy & Difficult & Essentially impossible & Very difficult & Impossible & Impossible \\
\hline Partial differential & Difficult & Essentially impossible & Impossible & Impossible & Impossible & Impossible \\
\hline
\end{tabular}

systems because, due to the strength of the real-world interactions between these components, failing to link them can cause severe misperceptions and indeed policy failures (Costanza 1987). Because reductionist thinking fails in the quest to understand complex systems, new concepts and methods must be devised.

Achieving a comprehensive understanding that is useful for modeling and prediction of linked ecological economic systems requires the synthesis and integration of several different conceptual frames. As Levins (1966) has described this search for robustness, "we attempt to treat the same problem with several alternative models each with different simplifications... Then, if these models, despite their different assumptions, lead to similar results we have what we call a robust theorem which is relatively free of the details of the model. Hence our truth is the intersection of independent lies" (p. 423).

Existing modeling approaches can be classified according to a number of criteria, including scale, resolution, generality, realism, and precision. The most useful approach within this spectrum of characteristics depends on the specific goals of the modeling exercise. We describe here a few examples of how one might match model characteristics with several of the possible modeling goals relevant for ecological economic systems, and we claim that a better appreciation of the range of possible model characteristics and goals can help to match characteristics and goals.

Complex-systems analysis offers great potential for generating insights into the behavior of linked ecological economic systems. These insights will be needed to change the behavior of the human population toward a sus- tainable pattern, one that works in synergy with the life-supporting ecosystems on which it depends. The next step in the evolution of ecological economic models is to fully integrate the two fields and not just transfer methods between them. Clark's (1976, 1981, 1985) bioeconomics work was the start of this recognition of the importance of linking the mutually interacting subparts. But much work remains to be done to bring the two fields and the technology that supports them to the point where their models can adequately interact. Transdisciplinary collaboration and cooperative synthesis among natural and social scientists will be essential (Norgaard 1989).

\section{Computers and modeling}

Until computers became available, the equations that described the dynamics of systems had to be solved analytically, severely limiting the level of complexity (as well as the resolution) of the systems that could be studied and the complexity of the dynamics that could be examined for any particular system. Table 1 shows the limits of analytical methods in solving various classes of mathematical problems in general.

Only relatively simple linear systems of algebraic or differential equations can, in general, be solved analytically. The problem is that most complex, living systems (like economies and ecosystems) are decidedly nonlinear, and efforts to approximate their dynamics with linear equations have been of only limited usefulness. In addition, complex systems often exhibit discontinuous and chaotic behavior (Rosser 1991) that can only be adequately represented with numerical methods and simulations using computers.

We differentiate here between the use of linear systems of equations to model complex-system dynamics (which does not work well) versus the use of linear systems to understand system structure (which may work reasonably well). Integrating these views of structure and dynamics is a key item for research on complex ecological economic systems.

In recent years, computers have become not only faster but also much more accessible. This ease of access has allowed researchers to develop methods to allow adaptive, evolutionary, dynamic solutions. For example, Holland and Miller (1991) describe how recent computer and machine learning (a form of artificial intelligence) advances have spawned "artificial adaptive agents," computer programs that can simulate evolution and acquire sophisticated behavioral patterns. In these programs, individual agents (e.g., processes, elements, and pieces of computer code) in networks of interacting agents reproduce themselves in the next time period based on some measure of their performance in the current time period. The system exhibits changing group behavior over time and mimics evolution. To exhibit this adaptive behavior, the actions of the agents must be assigned values, and the agents must act to increase these values over time. Algorithms like these can provide a realistic representation of ecological and economic processes.

Another useful technique is metamodeling, in which more general models are developed from detailed ones. Richard Cabe, Jason Shogren, and their colleagues (1991) have developed this technique to link models of agricultural production and economic behavior that could not nor- 
mally be used together because, for one, they run at different time and space scales. Their models, which cover the entire midwestern farm belt of the United States, provide a method for a quick and cost-efficient evaluation of ecological economic policies.

Computer hardware advances such as CRAY supercomputers and Connection Machines (massively parallel supercomputers) facilitate the modeling of complex systems using advanced numerical computation algorithms (e.g., finite difference and finite element routines, cellular automata algorithms, and emerging methods that employ at least a modicum of artificial intelligence). For example, parallel computers make high spatial resolution and regional and global ecological economic models computationally feasible (Costanza et al. 1990, Costanza and Maxwell 1991) and allow the types and resolution of evolutionary and metamodeling approaches to expand dramatically. These new capabilities, linked with a more realistic and pluralistic view of the various roles and limitations of models in understanding and decision making, can dramatically increase the effectiveness of modeling.

\section{Purposes of models}

Models are analogous to maps. Like maps, they have many possible purposes and uses, and no one map or model is right for the entire range of uses (Levins 1966, Robinson 1991). It is inappropriate to think of models or maps as anything but crude, although in many cases absolutely essential, abstract representations of complex territory. Their usefulness can best be judged by their ability to help solve the navigational problems faced. Models are essential for policy evaluation, but they are often also misused because there is "the tendency to use such models as a means of legitimizing rather than informing policy decisions. By cloaking a policy decision in the ostensibly neutral aura of scientific forecasting, policy-makers can deflect attention from the normative nature of that decision..." (Robinson in press).

In the case of modeling ecological economic systems, purposes can range from developing simple conceptual models to provide a general under- standing of system behavior, to detailed realistic applications aimed at evaluating specific policy proposals. It is inappropriate to judge this whole range of models by the same criteria. At minimum, the three criteria of realism (simulating system behavior in a qualitatively realistic way), precision (simulating behavior in a quantitatively precise way), and generality (representing a broad range of systems' behaviors with the same model) are necessary. Holling (1964) first described the fundamental trade-offs in modeling among these three criteria. Later, Holling (1966) and Levins (1966) expanded and further applied this classification. No single model can maximize all three of these goals, and the choice of which objectives to pursue depends on the fundamental purposes of the model. Several examples in the literature of ecological and economic models demonstrate the various ways in which trade-offs are made among realism, precision, and generality.

High-generality conceptual models. In striving for generality, models must give up some realism and/or precision. They can simplify relationships and/or reduce resolution. Simple linear and nonlinear economic and ecological models tend to have high generality but low realism and low precision (Brown and Swierzbinski 1985, Clark and Monroe 1975, Kaitala and Pohjola 1988, Lines 1989, 1990b). Examples include Holling's four-box model (Holling 1987), the ecological economy model of Brown and Roughgarden (1992), most conceptual macroeconomic models (Keynes 1936, Lucas 1975), economic growth models (Solow 1956), and the evolutionary games approach. For example, the ecological economy model (Brown and Roughgarden 1992) contains only three state variables (labor, capital, and natural resources), and the relationships among these variables are highly idealized. But the purpose of the model was not high realism or precision but rather to address some basic questions about the limits of economic systems in the context of their dependence on an ecological life-support base.

High-precision analytical models. Often one wants high precision (quan- titative correspondence between data and model) and is willing to sacrifice realism and generality. One strategy here is to keep resolution high but to simplify relationships and deal with short time frames. Some models strive to strike a balance between mechanistic small-scale models that trace small fluctuations in a system and more general whole-system approaches that remove some of the noise from the signal but do not allow the modeler to trace the source of system changes. The alternative some ecologists have devised is to identify one or a few properties that characterize the system as a whole (Wulff and Ulanowicz 1989). For example, Hannon and Joiris (1987) used an economic input-output model to examine relationships between biotic and abiotic stocks in a marine ecosystem; they found that this method allowed them to show the direct and indirect connection of any species to any other and to the external environment in this system at high precision (but low generality and realism). Also using input-output techniques, Duchin's $(1988,1992)$ aim was to direct development of industrial production systems to efficiently reduce and recycle waste in the manner of ecological systems. Large econometric models (Klein 1971) used for predicting short-run behavior of the economy belong to this class of models, because they are constructed to fit existing data as closely as possible, at the sacrifice of generality and realism.

High-realism impact-analysis models. When the goal is to develop realistic assessments of the behavior of specific complex systems, generality and precision must be relaxed. High-realism models are concerned with accurately representing the underlying processes in a specific system, rather than with precisely matching quantitative behavior or being generally applicable. Dynamic, nonlinear, evolutionary systems models at moderate to high resolution generally fall into this category. Coastal physical-biological-chemical models (Wroblewski and Hofmann 1989), which are used to investigate nutrient fluxes and contain large amounts of site-specific data, fall into this category, as do micromodels of behavior of particular business activities. Another example is a model of coastal landscape dynamics (Costanza 
et al. 1990), which includes high spatial and temporal resolution and complex nonlinear process dynamics. This model divides a coastal landscape into 1-square-kilometer cells, each of which contains a process-based dynamic ecological simulation model. Flows of water, sediments, nutrients, and biomass from cell to cell across the landscape are linked with internal ecosystem dynamics to simulate long-term successional processes and responses to various human impacts in a realistic way. But the model is site specific and of only moderate numerical precision.

Moderate-generality and moderateprecision indicator models. In many types of systems modeling, the desired outcome is to accurately determine the overall magnitude and direction of change, trading off realism for some moderate amount of generality and precision. For example, aggregate measures of system performance such as standard gross national product, environmentally adjusted net national product (or green NNP), which includes environmental costs (Mäler 1991), and indicators of ecosystem health (Costanza et al. 1992) fit into this category. The microcosm systems employed by Taub (1989) allow some standardization for testing ecosystem responses and developing ecosystem performance indices. Taub (1987) notes, however, that many existing indicators of change in ecosystems are based on implicit ecological assumptions that have not been critically tested, either for their generality, realism, or precision.

\section{Scale and hierarchy}

In modeling complex systems, the issues of scale and hierarchy are central (O'Neill et al. 1989). Some claim that the natural world, the human species included, contains a convenient hierarchy of scales based on interactionminimizing boundaries: scales ranging from atoms to molecules to cells to organs to organisms to populations to communities to ecosystems (including economic and/or human-dominated ecosystems) to bioregions to the global system and beyond (Allen and Starr 1982, O’Neill et al. 1986). By studying the similarities and differences among different kinds of sys- tems at different scales and resolutions, one might develop hypotheses and test them against other systems to explore their degree of generality and predictability.

The term scale in this context refers to both the resolution (spatial grain size, time step, or degree of complication of the model) and extent (in time, space, and number of components modeled) of the analysis. The process of scaling refers to the application of information or models developed at one scale to problems at other scales. In both ecology and economics, primary information and measurements are generally collected at relatively small scales (i.e., small plots in ecology or individuals or single firms in economics), and that information is then often used to build models at radically different scales (i.e., regional, national, or global). The process of scaling is directly tied to the problem of aggregation (the process of adding or otherwise combining components), which in complex, nonlinear, discontinuous systems (like ecological and economic systems) is far from a trivial problem (O'Neill and Rust 1979, Rastetter et al. 1992). For example, in applied economics, basic data sets are usually derived from national accounts that contain data that are linearly aggregated over individuals, companies, or organizations. Sonnenschein (1974) and Debreu (1974) have shown that, unless one makes strong and unrealistic assumptions about the individual units, the aggregate (largescale) relations between variables have no resemblance to the corresponding relations on the smaller scale.

Rastetter et al. (1992) describe and compare three basic methods for scaling that are applicable to complex systems. All of their methods are attempts to use information about the nonlinear small-scale variability in the large-scale models. They list partial transformations of the fine-scale mathematical relationships to coarse scale using a statistical expectations operator that incorporates the fine-scale variability; partitioning or subdividing the system into smaller, more homogeneous parts (i.e., spatially explicit modeling); and calibration of the fine-scale relationships to coarsescale data when this data is available. They go on to suggest a combination of these methods as the most effective overall method of scaling in complex systems.

A primary reason for aggregation error in scaling complex systems is the nonlinear variability in the fine-scale phenomenon. For example, Rastetter et al. (1992) give a detailed example of scaling a relationship for individual leaf photosynthesis as a function of radiation and leaf efficiency to estimate the productivity of the entire forest canopy. Because of nonlinear variability in the way individual leaves process light energy, one introduces significant aggregation error by simply using the fine-scale relationships among photosynthesis, radiation, and efficiency along with the average values for the entire forest to get total forest productivity.

One must somehow understand and incorporate this nonlinear fine-scale variability into the coarse-scale equations using some combination of the three methods mentioned above. The statistical expectations method implies deriving new coarse-scale equations that incorporate the fine-scale variability. The problem is that incorporation of this variability often leads to equations that are extremely complex and cumbersome (Rastetter et al. 1992). The partitioning method implies subdividing the forest into many relatively more homogeneous levels or zones and applying the basic finescale equations for each partition. This approach requires a method for adjusting the parameters for each partition, a choice of the number of partitions (the resolution), and an understanding of the effects of the choice of resolution and parameters on the results. The recalibration method implies simply recalibrating the fine-scale equations to coarse-scale data. It presupposes that coarse-scale data are available (as more than simply the aggregation of fine-scale data). In many important cases, however, this coarse-scale data is either extremely limited or is not available. Thus, although a judicious application of all three aggregation methods is necessary, from the perspective of complex systems modeling, the partitioning approach seems to hold particular promise, because it can take fullest advantage of emerging computer technologies and databases.

From the scaling perspective, hierarchy theory is a potentially useful 
tool for partitioning systems in ways that minimize aggregation error. According to hierarchy theory, nature can be partitioned into naturally occurring levels, which share similar time and space scales and which interact with higher and lower levels in systematic ways. Each level in the hierarchy experiences the higher levels as constraints and the lower levels as noise. For example, individual organisms experience the ecosystem they inhabit as a slowly changing set of constraints, and the operation of their component cells and organs is what matters most to them. However, Norton and Ulanowicz (1992) suggest that what appears to be noise at a lower level could be turned into significant perturbations on the higher level. This change can happen when a critical mass of components participate in a trend, a behavioral pattern, that affects the slower processes at the higher level. The rapid and extensive human uses of fossil fuels could be seen as such a trend, causing perturbations at the global atmospheric level, which might feed back and radically alter the framework of action at the lower level.

Shugart et al. (1991) explains the relationship between scales: "Clearly, natural patterns in environmental constraints contribute substantially to the spatial pattern and temporal dynamics of particular ecosystems...these patterns, especially temporal ones, may resonate with natural frequencies of plant growth forms (i.e., phenology and longevity) to amplify environmental patterns" (p. 232). The simplifying assumptions of hierarchy theory may ease the problem of scaling by providing a common (but somewhat generalized) set of rules that could be applied at any scale in the hierarchy.

\section{Fractals and chaos}

The concept of fractals (Mandelbrot 1977) can be seen as another related approach to the problem of scaling, based on the fundamental principle of self-similarity between scales. This concept implies a regular and predictable relationship between the scale of measurement (here meaning the resolution of measurement) and the measured phenomenon. For example, the measured length of a coastline is an increasing function of the resolution at which it is measured. At higher resolutions, one can recognize and measure more of the small-scale bays and indentations in the coast and the total length measured increases.

The relationship between length and resolution usually follows a regular pattern that can be summarized in the following equation:

$$
L=\mathrm{k} s^{(1-D)}
$$

where $L$ equals the length of the coastline or other fractal boundary, $s$ equals the size of the fundamental unit of measure or the resolution of the measurement, $k$ equals a scaling constant, and $D$ equals the fractal dimension.

Phenomena that fit this equation are said to be self-similar because, as resolution is increased, one perceives patterns at the smaller scale similar to those at the larger scale. This convenient scaling rule has proven useful in describing many kinds of complex boundaries and behaviors (Mandelbrot 1983, Milne 1991, Olsen and Schaffer 1990, Sugihara and May 1990, Turner et al. 1989). One test of the principle of self-similarity is that it can be applied to produce computergenerated shapes that have a decidedly natural and organic look to them (Mandelbrot 1977).

Certain nonlinear dynamical systems models exhibit behaviors whose phase plots $(x[t]$ versus $x[t-\mathrm{d} t])$ are fractals. These chaotic attractors, as they are called, are one of four possible pure types of attractors that can be used to classify system dynamics. The other three are point attractors (indicating stable, non-time varying behavior), periodic attractors (indicating periodic time behavior), and noisy attractors (indicating stochastic time behavior). Real-system behavior can be thought of as representing some combination of these four basic types.

The primary questions about the range of applicability of fractals and chaotic-systems dynamics to the practical problems of modeling ecological economic systems are the influence of scale, resolution, and hierarchy on the mix of behaviors one observes in systems. This problem is key for extrapolating from small-scale experiments or simple theoretical models to practical applied models of ecological economic systems.

\section{Resolution and predictability}

The significant effects of nonlinearities raise some interesting questions about the influence of resolution (including spatial, temporal, and component) on the performance of models, in particular on their predictability. For example, the relationship between the degree of complication (the number of components included) and the predictability of models is an important input to model design. Hofmann (1991) discusses this concern in the context of scaling coastal models to the global scale. The difficulty of using aggregate models that integrate over many details of finer resolution models is that the aggregated models may not be able to represent biological processes on the space and time scales necessary. Hofmann suggests that coupled detailed models (in which the output of one model becomes the input for another) may be a more practical method for scaling models to larger systems.

Costanza and Maxwell (in press) analyzed the relationship between spatial resolution and predictability and found that, although increasing resolution provides more descriptive information about the patterns in data, it also increases the difficulty of accurately modeling those patterns. There may be limits to the predictability of natural phenomenon at particular resolutions, and scaling rules that determine how both "data" and "model" predictability change with resolution.

Predictability (Colwell 1974) measures the reduction in uncertainty about one variable given knowledge of others using categorical data. One can define spatial autopredictability $\left(P_{a}\right)$ as the reduction in uncertainty about the state of a pixel in a scene, given knowledge of the state of adjacent pixels in that scene, and spatial cross-predictability $\left(P_{c}\right)$ as the reduction in uncertainty about the state of a pixel in a scene, given knowledge of the state of corresponding pixels in other scenes. $P_{a}$ is a measure of the internal pattern in the data, whereas $P_{c}$ is a measure of the ability of a model to represent that pattern.

Some limited testing of the relationship between resolution and predictability (by resampling land-use map data at different spatial resolutions) showed a strong linear relation- 
ship between the $\log$ of $P_{a}$ and the log of resolution (measured as the number of pixels per square kilometer). This fractal-like characteristic of selfsimilarity with decreasing resolution implies that predictability, like the length of a coastline, may be best described using a unitless dimension that summarizes how it changes with resolution. One can define a fractal predictability dimension $\left(D_{P}\right)$ in a manner analogous to the normal fractal dimension that summarizes this relationship. $D_{p}$ allows convenient scaling of predictability measurements taken at one resolution to other resolutions.

Cross-predictability $\left(P_{c}\right)$ can be used for pattern matching and testing the fit between map scenes. In this sense, it relates to the predictability of models versus the internal predictability in the data revealed by $P_{a}$. Although $P_{a}$ generally increases with increasing resolution (because more information is being included), $P_{c}$ generally falls or remains stable (because it is easier to model aggregate results than fine-grain ones). Thus, we can define an optimal resolution for a particular modeling problem that balances the benefit in terms of increasing data predictability $\left(P_{a}\right)$ as one increases resolution, with the cost of decreasing model predictability $\left(P_{c}\right)$. Figure 1 shows this relationship in generalized form.

These results may be generalizable to all forms of resolution (spatial, temporal, and number of components) and may shed some light on chaotic behavior in systems. When looking across resolutions, chaos may be the low level of model predictability that occurs as a natural consequence of high resolution. Lowering model resolution can increase model predictability by averaging out some of the chaotic behavior, at the expense of losing detail about the phenomenon. For example, Sugihara and May (1990) found chaotic dynamics for measles epidemics at the level of individual cities, but more predictable periodic dynamics for whole nations.

\section{Evolutionary approaches}

In modeling the dynamics of complex systems, it is impossible to ignore the discontinuities and surprises that often characterize these systems and the fact that they operate far from equi-

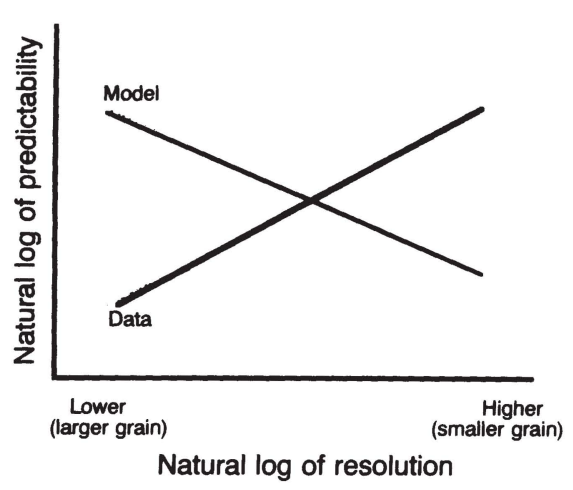

Figure 1. Hypothetical relationship between resolution and predictability of data and models, plotted on log-log axes (from Costanza and Maxwell in press). Data predictibility is a measure of the internal pattern in the data (e.g., the degree to which the uncertainty about the state of landscape pixels is reduced by knowledge of the state of adjacent pixels in the same map). Model predictability is a measure of the correspondence between data and models (e.g., the degree to which the uncertainty about the state of pixels is reduced by knowledge of the corresponding state of pixels in a model of the system). In general, data predictability rises with increasing resolution (because more internal patterns are perceived), whereas model predictability falls (because it becomes more difficult to match the high-resolution patterns). Particular types of models and data sets would fall on different lines, and certain types of models would require certain types of data. An optimal resolution occurs where the data and model predictability lines intersect.

librium in a state of constant adaptation to changing conditions (Holland and Miller 1991, Kay 1991, Lines 1990, Rosser 1991, 1992). The paradigm of evolution has been broadly applied to both ecological and economic systems (Arthur 1988, Boulding 1981, Lindgren 1991, Maxwell and Costanza in press) as a way of formalizing understanding of adaptation and learning behaviors in nonequilibrium dynamic systems. The general evolutionary paradigm posits a mechanism for adaptation and learning in complex systems at any scale using three basic interacting processes: information storage and transmission, generation of new alternatives, and selection of superior alternatives according to some performance criteria.

The evolutionary paradigm is different from the conventional optimi- zation paradigm popular in economics in at least four important respects (Arthur 1988): evolution is path dependent, meaning that the detailed history and dynamics of the system are important; evolution can achieve multiple equilibria; there is no guarantee that optimal efficiency or any other optimal performance will be achieved, due in part to path dependence and sensitivity to perturbations; and lock-in (survival of the first rather than survival of the fittest) is possible under conditions of increasing returns. Arthur (1988) notes, "conventional economic theory is built largely on the assumption of diminishing returns on the margin (local negative feedbacks)," but life itself can be characterized as a positive feedback, self-reinforcing, autocatalytic process (Günther and Folke in press, Kay 1991), and we should expect increasing returns, lockin, path dependence, multiple equilibria, and suboptimal efficiency to be the rule rather than the exception in economic and ecological systems.

\section{Cultural versus genetic evolution}

In biological evolution, the information storage medium is the genes, the generation of new alternatives is by sexual recombination or genetic mutation, and selection is performed by nature according to criteria of fitness based on reproductive success. The same process of change occurs in ecological, economic, and cultural systems, but the elements on which the process works are different. For example, in cultural evolution the storage medium is the culture (the oral tradition, books, film, or other storage medium for passing on behavioral norms), the generation of new alternatives is through innovation by individual members or groups in the culture, and selection is again based on the reproductive success of the alternatives generated. Reproduction is carried out by the spread and copying of the behavior through the culture rather than by biological reproduction.

One may also talk of economic evolution, a subset of cultural evolution dealing with the generation, storage, and selection of alternative ways of producing things and allocating that which is produced. The field of 
evolutionary economics has grown up in approximately the last decade based on these ideas (cf. Day 1989, Day and Groves 1975). Evolutionary theories in economics have already been successfully applied to problems of technical change, to the development of new institutions, and to the evolution of means of payment.

For large, slow-growing animals like humans, genetic evolution has a built-in bias toward the long run. Changing the genetic structure of a species requires that characteristics (phenotypes) be selected and accumulated by differential reproductive success. Behaviors learned or acquired during the lifetime of an individual cannot be passed on genetically. Genetic evolution is therefore usually a relatively slow process requiring many generations to significantly alter a species' physical and biological characteristics.

Cultural evolution is potentially much faster. Technical change is perhaps the most important and fastestevolving cultural process. Learned behaviors that are successful, at least in the short term, can be almost immediately spread to other members of the culture and passed on in the oral, written, or video record. The increased speed of adaptation that this process allows has been largely responsible for Homo sapiens' amazing success at appropriating the resources of the planet. Vitousek et al. (1986) estimate that humans directly control from $25 \%$ to $40 \%$ of the total primary production of the planet's biosphere, and this control is beginning to have significant effects on the biosphere, including changes in global climate and in the planet's protective ozone shield.

The costs of this rapid cultural evolution, therefore, are potentially significant. Like a car that has increased speed, humans are in more danger of running off the road or over a cliff. Cultural evolution lacks the built-in long-run bias of genetic evolution and is susceptible to being led by its hyperefficient short-run adaptability over a cliff into the abyss.

Another major difference between cultural and genetic evolution may serve as a countervailing bias, however. As Arrow (1962) has pointed out, cultural and economic evolution, unlike genetic evolution, can to some extent employ foresight. If society can see the cliff, perhaps it can be avoided.

Although market forces drive adaptive mechanisms (Kaitala and Pohjola 1988), the systems that evolve are not necessarily optimal, so the question remains: What external influences are needed, and when should they be applied to achieve an optimal economic system via evolutionary adaptation? The challenge faced by ecological economic systems modelers is to first apply the models to gain foresight and then to respond to and manage the system feedbacks in a way that helps avoid any foreseen cliffs (Berkes and Folke in press). Devising policy instruments and identifying incentives that can translate this foresight into effective modifications of the shortrun evolutionary dynamics is the challenge (Costanza 1987).

\section{Evolutionary criteria}

A critical problem in applying the evolutionary paradigm in dynamic models is defining the selection criteria a priori. In its basic form, the theory of evolution is circular and descriptive (Holling 1987). Those species or cultural institutions or economic activities survive that are the most successful at reproducing themselves. But we only know which ones were more successful after the fact. To use the evolutionary paradigm in modeling, we require a quantitative measure of fitness (or more generally performance) to drive the selection process.

Several candidates have been proposed for this function in various systems, ranging from expected economic utility to thermodynamic potential. Thermodynamic potential is interesting as a performance criterion in complex systems because even simple chemical systems can be seen to evolve complex nonequilibrium structures using this criterion (Nicolis and Prigogine 1977, 1989, Prigogine 1972), and all systems are (at minimum) thermodynamic systems (in addition to their other characteristics). Therefore, thermodynamic constraints and principles are applicable across both ecological and economic systems (Eriksson 1991).

This application of the evolutionary paradigm to thermodynamic systems has led to the development of far-from-equilibrium thermodynam- ics and the concept of dissipative structures (Prigogine 1972). An important research question is to determine the range of applicability of these principles and their appropriate use in modeling ecological economic systems.

Many dissipative structures follow complicated transient motions. Schneider and Kay (in press) propose a way to analyze these chaotic behaviors and note that, "Away from equilibrium, highly ordered stable complex systems can emerge, develop and grow at the expense of more disorder at higher levels in the system's hierarchy." It has been suggested that the integrity of far-from-equilibrium systems has to do with the ability of the system to attain and maintain its (set of) optimal operating point(s) (Kay 1991). The optimal operating point(s) reflect a state where self-organizing thermodynamic forces and disorganizing forces of environmental change are balanced. This idea has been elaborated and described as "evolution at the edge of chaos" by Bak and Chen (1991) and Kauffman and Johnson (1991).

The concept that a system may evolve through a sequence of stable and unstable stages leading to the formation of new structures seems well suited to ecological economic systems. For example, Gallopin (1989) stresses that to understand the processes of economic impoverishment "The focus must necessarily shift from the static concept of poverty to the dynamic processes of impoverishment and sustainable development within a context of permanent change. The dimensions of poverty cannot any longer be reduced to only the economic or material conditions of living; the capacity to respond to changes, and the vulnerability of the social groups and ecological systems to change become central" (p. 394).

In a similar fashion, Robinson (1991) argues that sustainability calls for maintenance of the dynamic capacity to respond adaptively, which implies that we should focus more on basic natural and social processes than on the particular forms these processes take at any time. Berkes and Folke (in press) have discussed the capacity to respond to changes in ecological economic systems, in terms of institution building, collective actions, 


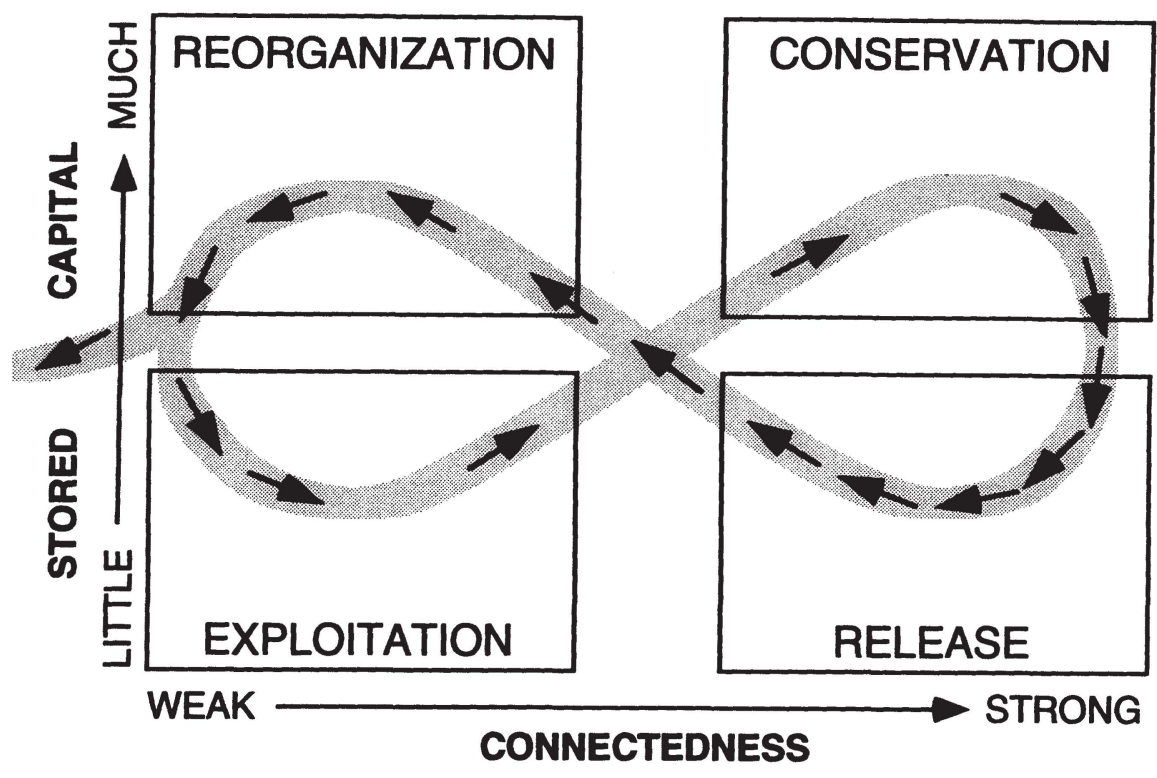

Figure 2. The four general system functions and the flow of events between them (from Holling 1987, 1992). The arrows show the speed of that flow in the ecosystem cycle; arrows close to each other indicate a rapidly changing situation and arrows far from each other indicate a slowly changing situation. The cycle reflects changes in two attributes: on the $\mathrm{Y}$ axis, the amount of accumulated capital (nutrients and carbon) stored in variables that are dominant keystone variables at the moment, and, on the $\mathrm{X}$ axis, the degree of connectedness among variables. The exit from the cycle indicated at the left of the figure indicates the stage where a flip is most likely into a less- or more-productive and organized system, that is, devolution or evolution as revolution.

cooperation, and social learning. These activities might enhance the capacity for resilience (increase the capacity to recover from disturbance) in interconnected ecological economic systems.

\section{The Holling model}

One broad conceptual application of these ideas to ecological and economic systems, with the goal of maximal generality, is the model of Holling (1987, 1992). Holling proposes four basic functions common to all complex systems and a spiraling evolutionary path through them (Figure 2). The functions (boxes) are: exploitation (e.g., r-strategists, pioneers, opportunists, and entrepreneurs), conservation (e.g., K-strategists, climax ecosystems, consolidation, and rigid bureaucracies), release (e.g., fire, storms, pests, and political upheavals), and reorganization (e.g., accessible nutrients and abundant natural resources). Within this model, systems evolve from the rapid colonization and exploitation phase, during which they capture easily accessible resources, to the conservation stage of building and storing increasingly complex structures. Examples of the exploitation phase are early successional ecosystems colonizing disturbed sites or pioneer societies colonizing new territories. Examples of the conservation phase are climax ecosystems or large, mature bureaucracies.

The release or "creative destruction" (Schumpeter 1950) phase represents the breakdown of mature structures via aperiodic events such as fire, storms, pests, or political upheavals. The released structure is then available for reorganization and uptake in the exploitation phase. The amount of ongoing creative destruction that takes place in the system is critical to its behavior. The conservation phase can often build elaborate and tightly bound structures by severely limiting creative destruction (the former Soviet Union is a good example), but these structures become brittle and susceptible to massive and widespread destruction. If some moderate level of release is allowed to occur on a more routine basis, the destruction is on a smaller scale and leads to a more resilient system. It could be argued that patterns of behavior with moder- ate levels of ongoing creative destruction evolved in those local communities and human cultures that managed to survive for thousands of years or more.

Creative destruction, in terms of shocks or surprises, seems to be crucial for system resilience and integrity. Similarly, it has been argued that episodic events, such as the Chernobyl accident, the Rhine chemical spill, and the death of seals in the North Sea, are shocks to the social-cultural value system and may stimulate positive change toward more resilient ecological economic systems (Berkes and Folke in press).

Fire climax systems, such as the pine forests of Yellowstone National Park, are a good example of the range of possibilities for creative destruction. In its unmanaged state, Yellowstone burned over extensive areas relatively often, but the high fire frequency kept the amount of fuel insufficient to create extremely destructive fires. The more-frequent, small- to moderatesize fires released nutrients stored in the litter and supported a spurt of new growth without destroying all the old growth. On the other hand, when fires were suppressed and controlled, fuel built up to high levels and (because control and suppression are never perfect-remember the former Soviet Union), when the fire did come it wiped out much of the forest.

The Holling four-box model may serve as a minimal ecological economic model aimed at generality (at the expense of precision and realism). It raises some interesting questions about the relationships among diversity, stability, resilience, control, creativity, surprise, and evolution in ecological and economic systems that are ripe for further analyses.

\section{Evolutionary game theory}

Evolutionary game theory is the combination of traditional game theory and evolutionary models. The evolution of evolutionary game theory is itself quite interesting, because it relied on several interacting disciplines. Game theory began with von Neuman (1928) as a mathematical exercise for analyzing parlor games. It continued with von Neuman and Morgenstern (1944), who developed the theory for applications in economics. The cen- 
tral idea is that a game consists of a number of players who all act rationally (i.e., use the information available to select strategies that will maximize their expected payoff). The situation when all players have picked their optimal strategies and no one can expect to increase their payoff is called a Nash equilibrium. Conventional game theory with rational players has had an enormous impact on economics, and an increasing number of economic problems are being studied using its tools.

Game theory was imported into evolutionary biology (Maynard-Smith and Price 1973, Maynard-Smith 1979, 1982) to improve understanding of biological processes. There was, however, an important change in the interpretation. Whereas economists used static, rational strategic choices on the part of the players, Maynard-Smith introduced evolution by identifying strategies with genes and the payoff with reproductive success. Reproductive strategies with high payoffs would be expected to have a proportionally higher representation in the population. A Nash equilibrium corresponds to an evolutionarily stable strategy (i.e., a strategy that would be immune to invasion by other strategies).

After this further development within evolutionary biology, evolutionary game theory was then reimported into economics when it became clear that it could be used for an improved understanding of the evolution of various economic institutions (e.g., means of payments and property rights) and of technical processes in production. Economists interested in evolutionary game theory have even started reapplying it to biological evolution (Selten 1980), and political scientists and others have developed it for the interdisciplinary analysis of the evolution of cooperation in both economic and ecological systems (Axelrod 1984).

Through research within four disciplines-mathematics, biology, economics, and political science-evolutionary game theory has developed as a rather important tool for understanding these social and biological processes in isolation. To address ecological economic systems, conventional game theory must be integrated with evolutionary game theory. This integration would include the analysis of games in which different subsets of the players have different time horizons, payoff structures, and objectives. For example, some organisms within the system may choose strategies not only with regard to reproductive success but also with regard to other goals. Some progress has been made along these lines (e.g., Banerjee and Weibull 1991), and it seems to be a fruitful area for future research.

\section{Summary of questions and opportunities}

Based on this synthesis, the major researchable questions and opportunities in modeling complex ecological economic systems can be divided into three broad, interdependent categories. These categories are listed below.

Application of the evolutionary paradigm to modeling ecological economic systems. The evolutionary paradigm provides a general framework for complex ecological economic systems dynamics. It incorporates the elements of uncertainty, surprise, learning, path dependence, multiple equilibria, suboptimal performance, lock-in, and thermodynamic constraints. In applying the evolutionary paradigm, a key feature is the choice of the measure (or multiple measures) of performance on which the system's selection process will work. Several such measures have been proposed and partially tested, but additional research and testing in this area may have a high payoff. An important research question is the range of applicability of nonequilibrium thermodynamic principles and their appropriate use in modeling ecological economic systems. Key methods include adaptive computer simulation models and integrated conventional/evolutionary game theory.

Scale and hierarchy considerations in modeling ecological economic systems. The key questions involve exactly how hierarchical levels interact with each other and how to further develop the three basic methods of scaling (statistical expectations, partitioning, and recalibration) for application to complex ecological economic systems. Additional questions concern the range of applicability of fractals and chaotic-systems dynamics to the practical problems of modeling ecological economic systems. In particular, what is the influence of scale, resolution, and hierarchy on the mix of behaviors one observes in systems? This question is key for extrapolating from small-scale experiments or simple theoretical models to practical applied models of ecological economic systems at regional and global scales.

The nature and limits of predictability in modeling ecological economic systems. The significant effects of nonlinearities raise some interesting questions about the influence of resolution (including spatial, temporal, and component) on the performance of models, in particular on their predictability. There may be limits to the predictability of natural phenomena at particular resolutions, and fractallike rules that determine how both data and model predictability change with resolution. To test these limits, we need better measures of model correspondence with reality and longterm, aggregate-system performance that incorporate the three conflicting criteria of generality, realism, and precision.

\section{Acknowledgments}

The Beijer International Institute for Ecological Economics, a research institute of the Royal Swedish Academy of Sciences, provided support for the preparation of this article, which was presented at the workshop on Complex Ecological Economic Systems Modeling held at the Beijer Institute in Stockholm, Sweden, 27-29 July 1992. We thank C. S. Holling, J. Kay, R. E. Ulanowicz, J. Bartholomew, and four anonymous reviewers for detailed comments and suggestions on earlier drafts. We are also indebted to the other participants at the Beijer workshop (G. Brown, C. Clark, P. Dasgupta, F. Duchin, K.-E. Eriksson, S. O. Funtowicz, G. Gallopin, C. Lindgrend, B. Hannon, E. Hofmann, H. Isomäki, R. Kaufmann, V. Kaitala, M. Lines, T. Maxwell, J. McGlade, J. Robinson, C. Perrings, J. B. Rosser, J. Roughgarden, J. Shogren, and F. Taub) for their valuable input and feedback on the article. The article also benefited from interactions with faculty from the University of Maryland's 
Multiscale Experimental Ecosystem Research Center, which is devoted to research on many of the topics we have identified.

\section{References cited}

Allen, T. F. H., and T. B. Starr. 1982. Hierarchy. University of Chicago Press, Chicago.

Arrow, K. 1962. The economic implications of learning by doing. Review of Economic Studies. 29: 155-173.

Arthur, W. B. 1988. Self-reinforcing mechanisms in economics. Pages 9-31 in P. W. Anderson, K. J. Arrow, and D. Pines, eds. The Economy as an Evolving Complex System. Addisson-Wesley, Redwood City, CA.

Axelrod, R. 1984. Evolution of Cooperation. Basic Books, New York.

Bak, P., and K. Chen. 1991. Self-organized criticality. Sci. Am. 264: 46.

Banerjee, A., and J. W. Weibull. 1991. Evolutionary selection and rational behavior. Research Papers in Economics 4, Department of Economics, University of Stockholm, Sweden.

Berkes, F., and C. Folke. In press. Investing in cultural capital for a sustainable use of natural capital. In A. M. Jansson, C. Folke, R. Costanza, and M. Hammer, eds. Investing in Natural Capital: The Ecological Economic Approach to Sustainability.

Boulding, K. E. 1981. Evolutionary Economics. Sage, Beverly Hills, CA.

Brown, G. M., and J. Roughgarden. 1992. An ecological economy: notes on harvest and growth. Beijer Discussion Paper Series 12, Beijer International Institute of Ecological Economics, Stockholm, Sweden.

Brown, G. M., and J. Swierzbinski. 1985. Endangered species, genetic capital and costreducing $R \& D$. Pages $111-127$ in $D$. O. Hall, N. Myers, and N. S. Margaris, eds. Economics of Ecosystems Management. Dr. W. Junk Publ., Dordrecht, The Netherlands.

Cabe, R., J. Shogren, A. Bouzaher, and A. Carriquiry. 1991. Metamodels, response functions, and research efficiency in ecological economics. Working paper 91-WP 79, Center for Agricultural and Rural Development, Iowa State University, Ames.

Clark, C. W. 1976. Mathematical Bioeconomics. John Wiley \& Sons, New York.

1981. Bioeconomics of the ocean. BioScience 31: 231-237.

.1985. Bioeconomic Modelling and Fisheries Management. John Wiley \& Sons, New York.

Clark, C. W., and G. R. Munro. 1975. The economics of fishing and modern capital theory: a simplified approach. J. Environ. Econ. Manage. 2: 92-106.

Colwell, R. K. 1974. Predictability, constancy, and contingency of periodic phenomena. Ecology 55: 1148-1153.

Costanza, R. 1987. Social traps and environmental policy. BioScience 37: 407-412.

Costanza, R., and T. Maxwell. 1991. Spatial ecosystem modeling using parallel processors. Ecol. Model. 58: 159-183

In press. Resolution and predictability: an approach to the scaling problem. Landscape Ecol.

Costanza, R., B. Norton, and B. J. Haskell, eds.
1992. Ecosystem Health: New Goals for Environmental Management. Island Press, Washington, DC.

Costanza, R., F. H. Sklar, and M. L. White. 1990. Modeling coastal landscape dynamics. BioScience 40: 91-107

Day, R. H. 1989. Dynamical systems, adaptation and economic evolution. MRG Working Paper no. M8908, University of Southern California, Los Angeles.

Day, R. H., and T. Groves, eds. 1975. Adaptive Economic Models. Academic, New York.

Debreu, G. 1974. Excess demand functions. Journal of Mathematical Economics 1: $15-23$.

Duchin, F. 1988. Analyzing structural change in the economy. Pages $113-128$ in $\mathrm{M}$. Ciaschini, ed. Input-Output Analysis: Current Developments. Chapman and Hall, New York.

.1992. Industrial input-output analysis: implications for industrial ecology. Proc. Natl. Acad. Sci. 89: 851-855.

Eriksson, K.-E. 1991. Physical foundations of ecological economics. Pages 186-196 in L. O. Hansson and B. Jungen, eds. Human Responsibility and Global Change. University of Güteborg Press, Güteborg, Sweden.

Gallopin, G. C. 1989. Global impoverishment, sustainable development and the environment: a conceptual approach. International Social Science Journal 121: 375-397.

Günther, F., and C. Folke. In press. Characteristics of nested living systems. Journal of Biological Systems.

Hannon, B., and C. Joiris. 1987. A seasonal analysis of the southern North Sea ecosystem. Ecology 70: 1916-1934.

Hofmann, E. E. 1991. How do we generalize coastal models to global scale? Pages $401-417$ in R. F. C. Mantoura, J. M. Martin, and R. Wollast, eds. Ocean Margin Processes in Global Change. John Wiley \& Sons, New York.

Holland, J. H., and J. H. Miller. 1991. Artificial adaptive agents in economic theory. American Economic Review 81: 365-370.

Holling, C. S. 1964. The analysis of complex population processes. Can. Entomol. 96: 335-347.

1966. The functional response of invertebrate predators to prey density. Mem. Entomol. Soc. Can. 48.

1987. Simplifying the complex: the paradigms of ecological function and structure. European Journal of Operational Research 30: 139-146.

.1992. Cross-scale morphology, geometry and dynamics of ecosystems. Ecol. Monogr. 62: 447-502

Kaitala, V., and M. Pohjola. 1988. Optimal recovery of a shared resource stock: a differential game model with efficient memory equilibria. Nat. Res. Model. 3: 91-119.

Kauffman, S. A., and S. Johnson. 1991. Coevolution to the edge of chaos: coupled fitness landscapes, poised states, and coevolutionary avalanches. J. Theor. Biol. 149:467-505.

Kay, J. J. 1991. A nonequilibrium thermodynamic framework for discussing ecosystem integrity. Environ. Manage. 15: 483-495.

Keynes, J. M. 1936. General Theory of Employment, Interest and Money. Harcourt Brace, London, UK.

Klein, L. R. 1971. Forecasting and policy evaluation using large-scale econometric models: the state of the art. Pages 133-177 in M. D. Intriligator, ed. Frontiers of Quantitative Economics. North-Holland Publ., Amsterdam, The Netherlands.

Levins, R. 1966. The strategy of model building in population biology. Am. Sci. 54:421-431.

Lindgren, K. 1991. Evolutionary phenomena in simple dynamics. Pages 295-312 in C. G. Langton, C. Taylor, J. D. Farmer, and S. Rasmussen. Artificial Life. Addison-Wesley, Redwood City, CA.

Lines, M. 1989. Environmental noise and nonlinear models: a simple macroeconomic example. Economic Notes 19: 376-394.

1990. Stochastic stability considerations: a nonlinear example. International Review of Economics and Business 37: 219-233.

Lucas, R. E. 1975. An equilibrium model of the business cycle. Journal of Political Economy 83: 1113-45.

Mäler, K.-G. 1991. National accounts and environmental resources. Environmental and Resource Economics 1: 1-15.

Mandelbrot, B. B. 1977. Fractals: Form, Chance and Dimension. W.H. Freeman, San Francisco, CA.

1983. The Fractal Geometry of $\mathrm{Na}$ ture. W.H. Freeman, San Francisco, CA.

Maxwell, T., and R. Costanza. In press. An approach to modelling the dynamics of evolutionary self-organization. Ecol. Model.

Maynard-Smith, J. 1979. Game theory and the evolution of behavior. Proc. R. Soc. Lond. B 205: 475-488.

1982. Evolution and the Theory of Games. Cambridge University Press, New York.

Maynard-Smith, J., and G. R. Price. 1973. The logic of animal conflict. Nature 246: 15-18

Milne, B. T. 1991. Lessons from applying fractal models to landscape patterns. Pages 199-235 in M. G. Turner and R. Gardner, eds. Quantitative Methods in Landscape Ecology. Springer-Verlag, New York.

Nicolis, G., and I. Prigogine. 1977. Self-organization in Non-equilibrium Systems. John Wiley \& Sons, New York.

1989. Exploring Complexity. W.H. Freeman, New York.

Norgaard, R. B. 1989. The case for methodological pluralism. Ecological Economics 1: 37-57.

Norton, B. G., and R. E. Ulanowicz. 1992. Scale and biodiversity policy: a hierarchical approach. Ambio 21: 244-249.

Olsen, L. F., and W. M. Schaffer. 1990. Chaos versus noisy periodicity: alternative hypotheses for childhood epidemics. Science 249: 499-504.

O’Neill, R. V., D. L. DeAngelis, J. B. Waide, and T. F. H. Allen. 1986. A Hierarchical Concept of Ecosystems. Princeton University Press, Princeton, NJ.

O'Neill, R. V., A. R. Johnson and A. W. King. 1989. A hierarchical framework for the analysis of scale. Landscape Ecol. 3: 193-205.

O’Neill, R. V., and B. Rust. 1979. Aggregation error in ecological models. Ecol. Model. 7: 91-105.

Prigogine, I. 1972. Thermodynamics of evolution. Physics Today 23: 23-28.

Rastetter, E. B., A. W. King, B. J. Cosby, G. M. Hornberger, R. V. O'Neill, and J. E. Hobbie. 
1992. Aggregating fine-scale ecological knowledge to model coarser-scale attributes of ecosystems. Ecological Applications 2: $55-70$.

Robinson, J. B. 1991. Modelling the interactions between human and natural systems. International Social Science Journal 130: 629-647.

Of maps and territories: the use and abuse of socio-economic modelling in support of decision-making. Technological Forecast and Social Change.

Rosser, J. B. 1991. From Catastrophe to Chaos: A General Theory of Economic Discontinuities. Kluwer, Amsterdam, The Netherlands.

1992. The dialogue between the economic and ecologic theories of evolution. Journal of Economic Behavior and Organization 17: 195-215.

Schneider, E. D., and J. J. Kay. In press. Life as a manifestation of the second law of thermodynamics. International Journal of Mathematical and Computer Modelling.

Schumpeter, J. A. 1950. Capitalism, Socialism and Democracy. Harper \& Row, New York.

Selten, R. 1980. A note on evolutionary stable strategies in asymmetrical animal conflicts. J. Theoret. Biol. 84: 93-101.

Shugart, H. H. 1989. The role of ecological models in long-term ecological studies. Pages 90-109 in G. E. Likens, ed. Long-Term Studies in Ecology: Approaches and Alternatives. Springer-Verlag, New York.

Shugart, H. H., G. B. Bonan, D. L. Urban, W. K. Lavenroth, W. J. Parton, and G. M. Hornberger. 1991. Computer models and long-term ecological research. Pages 221-239 in P. G. Risser, ed. Long-term Ecological Research: An International Perspective. John Wiley \& Sons, New York.

Solow, R. M. 1956. A contribution to the theory of economic growth. Quarterly Journal of Economics 70: 65-94.

Sonnenschein, H. 1974. Market excess demand functions. Econometrica 40: 549-563.

Sugihara, G., and R. M. May. 1990. Nonlinear forecasting as a way of distinguishing chaos from measurement error in time series. Nature 344: 734-741

Taub, F. B. 1987. Indicators of change in natural and human impacted ecosystems: status. Pages 115-144 in S. Draggan, J. J. Cohrssen, and R. E. Morrison, eds. Preserving Ecological Systems: The Agenda for Long-term Research and Development. Praeger, New York.

.1989. Standardized aquatic microcosm: development and testing. Pages 47-92 in A. Boudou and F. Ribeyre, eds. Aquatic Ecotoxicology: Fundamental Concepts and Methodologies. vol II. CRC Press, Boca Raton, FL.

Turner, M. G., R. Costanza, and F. H. Sklar. 1989. Methods to compare spatial patterns for landscape modeling and analysis. Ecol. Model. 48: 1-18.

Vitousek, P., P. R. Ehrlich, A. H. Ehrlich, and P. A. Matson. 1986. Human appropriation of the products of photosynthesis. BioScience 36: 368-373.

von Bertalanffy, L. 1968. General System Theory: Foundations, Development, Applications. George Braziller, New York.

von Neuman, J. 1928. Sur Theorie der Gesellschaftsspield. Mathematische Analen 100: 295-320.

von Neuman, J., and O. Morgenstern. 1944. Theory of Games and Economic Behavior. Princeton University Press, Princeton, NJ.

Wulff, F., and R. E. Ulanowicz. 1989. A comparative anatomy of the Baltic Sea and Chesapeake Bay ecosystems. Pages 232-256 in F. Wulff, J. G. Field, and K. H. Mann, eds. Network Analysis of Marine Ecology: Methods and Applications. Springer-Verlag, NY.

Wroblewski, J. S., and E. E. Hofmann. 1989. U.S. interdisciplinary modeling studies of coastal-offshore exchange processes: past and future. Prog. Oceanogr. 23: 65-99.

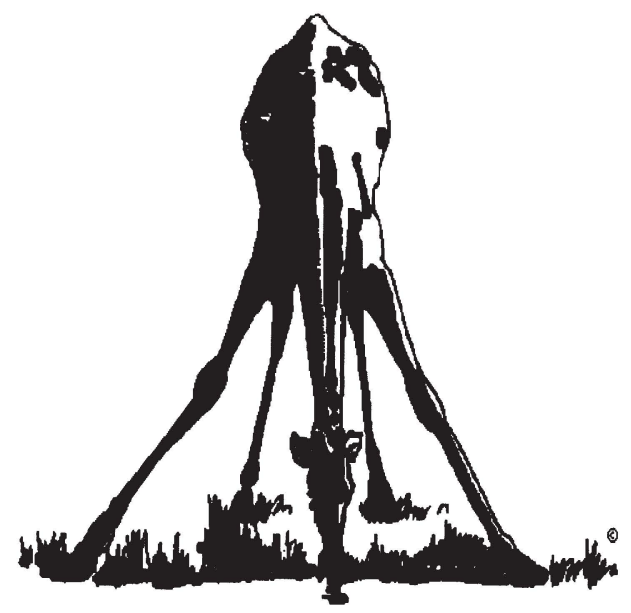

At bookstores or order toll-free

\section{1-800-822-6657. \\ University of California Press}

Berkeley Los Angeles

New York London

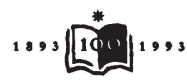

